\title{
Nano-Sculpting of Suspended CVD Graphene with Helium and Neon Ion Beams
}

\author{
D.A. Cullen, ${ }^{1}$ J. Swett, ${ }^{2}$ A. Rondinone, ${ }^{3}$ P. Bedworth, ${ }^{2}$ S. Heise, ${ }^{2}$ and S. Sinton ${ }^{2}$ \\ 1. Materials Science \& Technology Division, ORNL, Oak Ridge, TN 37831-6064 USA. \\ 2. Lockheed Martin Space Systems Co., Adv. Technology Center, Palo Alto, CA 94304 USA. \\ 3. Center for Nanophase Materials Sciences, ORNL, Oak Ridge, TN 37831-6493 USA.
}

The ability to precisely modify graphene is critical to a variety of application spaces, ranging from nanoscale electronics to chemical sensors [1,2]. The recent development and production of gas field ion source (GFIS) systems yields new opportunities for fabricating sub-10nm feature sizes within graphene sheets, as demonstrated by the etching of suspended graphene nanodevices with a helium ion beam [3]. However, the precision-controlled nanoscale-sculpting of graphene layers is challenging, as complex and interactive milling mechanisms, such as knock-on displacement, electronic interactions, and thermal processes, compete with self-healing and beam-induced deposition.

In the present study, we explore the fundamental principles and mechanisms for controllable nanosculpting (ion-milling using a Zeiss Orion NanoFab, Fig. 1a) of suspended graphene films produced by chemical vapor deposition (CVD). The impact of different aspects of the milling process, e.g., ion choice (helium or neon), beam energy, and ion dose, on pore creation and any associated defect formation, will be systematically explored (Fig. 1b). Ion milling efforts are coupled with low-voltage, aberration-corrected scanning transmission electron microscopy (STEM) in a Nion UltraSTEM 100 (Fig. 1c) to quantify the resulting pore size, lattice defects, and defect density, as shown in Fig. 1d-e. Careful measurements performed at various stages of milling (Fig. 2) will reveal any changes in defect density and carbon coordination with increasing ion dose, and explore aspects of electron-beam induced healing [4]. Changes in the density of single-vacancies, divacancies, multi-vacancies, lattice deformations (Frenkel Pairs and Stone-Wales defects), amorphitization, substitutions, and voids, will be discussed in the context of theoretical calculations and atomistic simulations [5].

All of the aforementioned experiments are being conducted on CVD graphene films, which have been robustly characterized by STEM prior to and after ion milling. Future paths towards meeting the ultimate goal of precise and repeatable sculpting of nano-scaled features in graphene will also be discussed [6].

References:

[1] Y Zhang et al, Nature 459 (2009) p. 820.

[2] F Schedin et al, Nature Materials 6 (2007) p. 652.

[3] MC Leme et al, ACS Nano, 3 (2009) p. 2674.

[4] R Zan et al, Nano Letters 12 (2012) p. 3936.

[5] O Lehtinen et al, Nanotechnology 22 (2011) p. 175306.

[6] Research supported by Independent Research \& Development funding at Lockheed Martin and through a user project supported by ORNL's Center for Nanophase Materials Sciences (CNMS), which is a Dept. of Energy, Office of Science User Facility. 

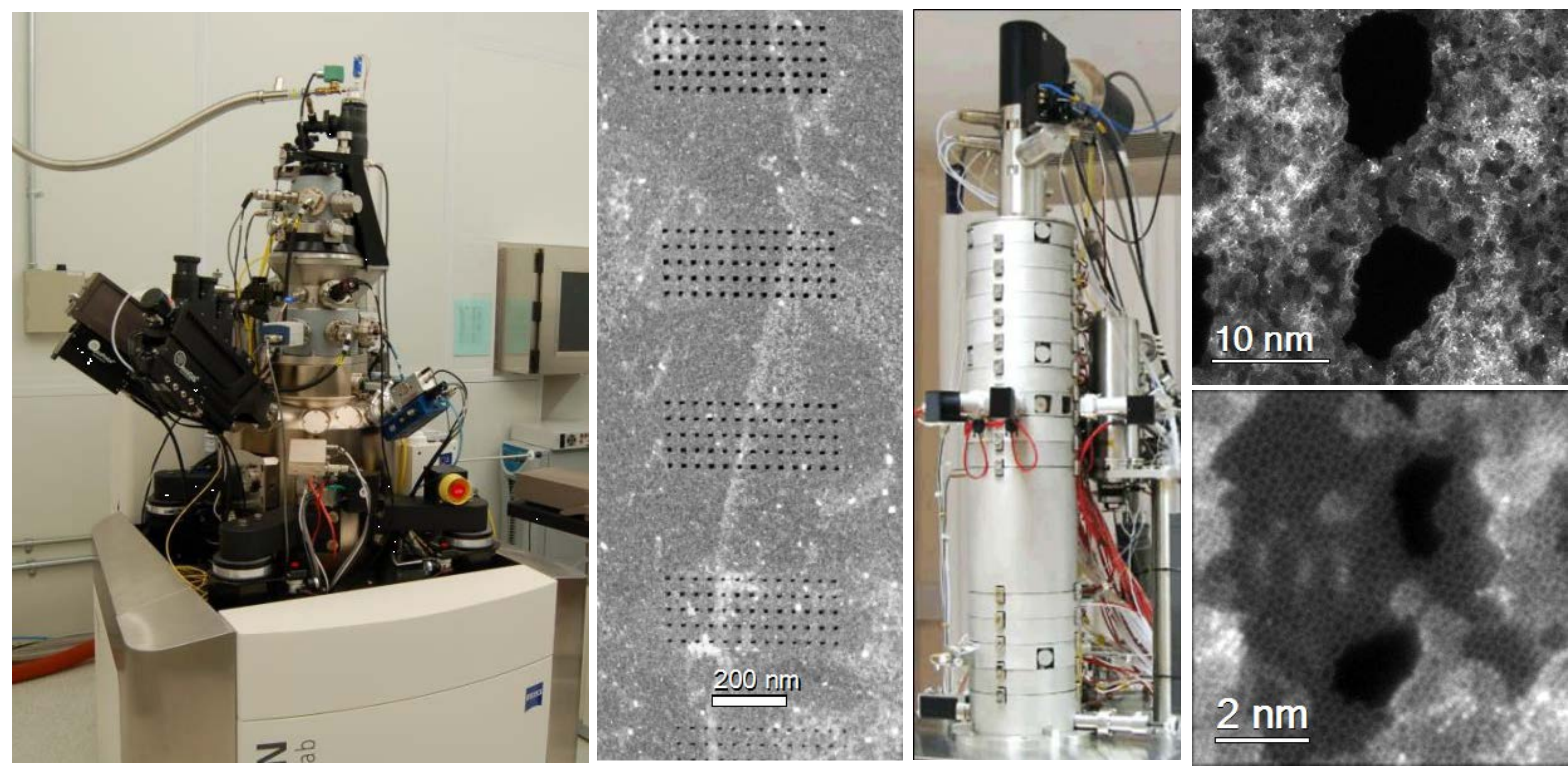

Figure 1. (a) Zeiss Orion NanoFab helium and neon ion microscope. (b) Pore arrays formed by helium ion milling. (c) Nion UltraSTEM 100 high-performance dedicated STEM. (d-e) Atomic-resolution STEM images of pores in graphene formed using a helium ion beam.
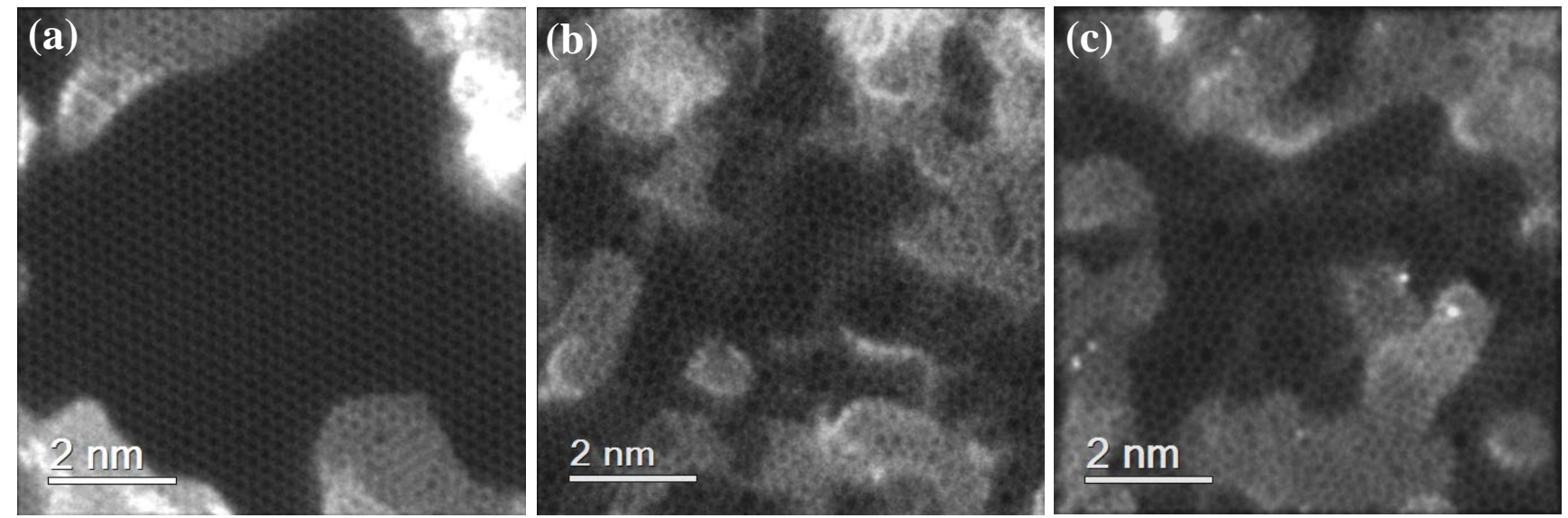

Figure 2. Example of the progression of pore formation in suspended CVD graphene produced via NanoFab milling, as imaged in the Nion UltraSTEM 100. (a) Pristine graphene, (b) intermediate He ion dose, and (c) high He ion dose. 